\title{
Curing metastatic testicular cancer
}

\author{
Lawrence H. Einhorn* \\ Indiana University Medical Center, 535 Barnhill Drive, RT 473, Indianapolis, IN 46202-5289 \\ Contributed by Lawrence H. Einhorn, February 5, 2002
}

This contribution is part of the special series of Inaugural Articles by members of the National Academy of Sciences elected on May $1,2001$.

Our initial studies with cisplatin + vinblastine + bleomycin began 27 years ago in 1974, changing the cure rate for disseminated disease from 5 to $60 \%$. Subsequently, through random prospective clinical trials, we have modified the treatment regimen to reduce both the duration and dosages of the chemotherapy drugs. Cisplatin + etoposide was first used at Indiana University as salvage chemotherapy in 1978, representing the first time that a solid tumor had been cured with second-line chemotherapy. We next did a clinical trial comparing bleomycin + etoposide + cisplatin (BEP) to cisplatin + vinblastine + bleomycin. The BEP regimen was proven to have less toxicity and a higher cure rate and therefore, since 1984, has been standard chemotherapy. More recent studies have evaluated the use of lesser chemotherapy to maintain the same cure rate for patients with good-prognosis disease. Standard therapy for these patients is either three courses of BEP or four courses of EP, and over $90 \%$ of these patients will be cured of their disease. Patients who are not cured with their initial BEP chemotherapy are usually treated with salvage chemotherapy. Approximately $50 \%$ of these testicular cancer patients will subsequently be cured with salvage chemotherapy with tandem transplant of high-dose chemotherapy with peripheral stem cell rescue. Testicular cancer has become a model for a curable neoplasm. In the early 1970s, metastatic testicular cancer was associated with only $5 \%$ survival. Today, with modern chemotherapy and surgery techniques, $\mathbf{8 0} \%$ of patients will survive their disease.

G erm cell tumors are relatively uncommon, accounting for only $1 \%$ of male malignancies in the United States. The highest worldwide incidence is in Scandinavian countries; by contrast, testicular cancer is rare in African Americans. The primary age group is 15-35 for nonseminomatous tumors and a decade older for seminoma.

In 2001 , there were $\approx 8,000$ newly diagnosed cases in the United States, contrasting sharply with the 190,000 cases of prostate cancer, the most common male malignancy.

Despite the paucity of cases, this tumor has become an extremely important oncological disease. First, it is the most common carcinoma in young men ages $15-35$ and thus has the potential to greatly shorten productive years of life. Second, available serum markers (alphafetoprotein and human chorionic gonadotropin) allow the clinician to make important and accurate treatment-related decisions. Third, testicular cancer has been a model for multidisciplinary care, as surgical resection of postchemotherapy radiographically persistent disease can improve the cure rate. Fourth, germ cell tumors have become an excellent testing ground for active experimental drugs (e.g., cisplatin, etoposide, and ifosfamide, all of which were approved by the Food and Drug Administration primarily on the basis of data in testicular cancer). The goal of chemotherapy in germ cell tumors is never merely palliation or prolongation of survival, but cure.

\section{Combination Chemotherapy and Clinical Trials}

Combination chemotherapy began to be used in patients with malignant diseases 30 years ago. The principle was that malignant clones of cells would rapidly develop drug resistance to a single cytolytic agent, thereby permitting disease progression and death after an initial clinical response. The strategies of combination chemotherapy, then and now, remain the same:

(i) use drugs with known single-agent activity;

(ii) use drugs with nonoverlapping toxicity. Because the major dose-limiting toxicity for most drugs is myelosuppression, nonmyelosuppressive antineoplastic agents are of particular value;

(iii) the cytolytic agents should have unique and separate mechanisms of action;

(iv) develop combinations demonstrating a synergistic rather than merely an additive effect.

Although there was some success with single antineoplastic agents such as cyclophosphamide for Burkitt's lymphoma, or methotrexate for gestational choriocarcinoma, the more dramatic improvements in survival and cure were the result of combination chemotherapy. Hematologic malignancies such as childhood acute lymphoblastic leukemia and Hodgkin's and non-Hodgkin's lymphoma were the initial beneficiaries of this approach. At about the same time (30 years ago), clinical trials became a powerful tool for hypothesis generation and proof of principle through random prospective phase III studies. This led to dramatic improvements in survival of pediatric cancers such as rhabdomyosarcoma, Ewing's sarcoma, osteosarcoma, etc.

\section{Early Chemotherapy Studies}

Before the usage of cisplatin combination chemotherapy, standard chemotherapy for disseminated testicular cancer consisted of dactinomycin, alone or in combination with methotrexate and chlorambucil. Thirty years ago, M. C. Li and colleagues at Memorial Sloan Kettering Hospital (New York) recognized that testis cancer was chemosensitive, with a $50 \%$ objective response rate including 10-20\% complete remission and a 5-10\% cure rate (1). M. L. Samuels and colleagues at M. D. Anderson Hospital (Houston) later evaluated vinblastine + bleomycin, a synergistic regimen in preclinical studies, and achieved a $25 \%$ long-term disease-free survival (2). This was a higher success rate than would have been predicted from the modest $5 \%$ cure rates of these drugs as single agents and appeared to validate the preclinical studies of this synergistic two-drug regimen. However, the drug that revolutionized the cure rate for patients with metastatic testicular cancer was cisplatin. Cisdiamminedichloroplatinum (cisplatin) was the first heavy metal to be evaluated as an anticancer therapy and resulted from the serendipitous finding and subsequent intellectual acumen of Barnett Rosenberg in the Department of Biophysics at Michigan State University (3). In 1973, cisplatin was first evaluated in early phase I-II clinical trials. Included among the patients in these studies were men with testicular cancer who had failed prior chemotherapy (usually dactinomycin) (4). There is enthusiasm when novel agents are capable of achieving a modest partial remission rate in refractory carcinomas. However, cisplatin attained not only three partial but also three complete remissions in 11 patients with refractory testicular cancer (4). Furthermore, despite initial severe nausea, vomiting, and nephrotoxicity, this

Abbreviations: PVB, cisplatin + vinblastine + bleomycin; BEP, etoposide + bleomycin; NED, no evidence of disease.

*E-mail: leinhorn@inpui.edu. 
drug was relatively nonmyelosuppressive, leading to inclusion as a component of combination chemotherapy. Subsequent improvements in supportive care have greatly mitigated the nephrotoxicity and emesis. This remains a dramatic example of the value, for patients and scientists, of clinical trials with novel experimental agents.

With this background, in August 1974, we began our initial cisplatin + vinblastine + bleomycin (PVB) study at Indiana University, using the established two-drug synergistic regimen of vinblastine + bleomycin and simply adding the then experimental promising drug cisplatin (5). The PVB regimen fulfilled the requirements for a successful combination chemotherapy regimen: single-agent activity for each component of the PVB regimen, different and unique mechanism of action for the three agents, separate and nonoverlapping toxicity, allowing administration of each drug in full dosage, and evidence of preclinical synergism (vinblastine + bleomycin).

\section{PVB Studies}

From 1974 to 1976, we initiated and completed our first PVB study (5). ${ }^{\dagger}$ As was traditional in the mid-1970s, induction therapy was followed by maintenance chemotherapy (vinblastine 0.3 $\mathrm{mg} / \mathrm{kg}$ monthly for a total of 2 years of chemotherapy), in an attempt to achieve total cell kill, as popularized with maintenance chemotherapy in childhood acute lymphoblastic leukemia. Four courses of PVB induction chemotherapy were used. This number was chosen because of concern (in 1974) of cumulative cisplatin-induced nephrotoxicity. We had a good early estimate of optimal duration of induction chemotherapy, as 25 years after completion of this original PVB trial, there is still no evidence to support the administration of more than four consecutive course of cisplatin combination chemotherapy.

Thirty-three of $47(70 \%)$ patients attained a complete remission, and an additional five patients $(11 \%)$ were rendered disease-free by postPVB surgical resection of radiographically persistent disease. Fifty-three percent of patients were cured with PVB, representing a 1-logarithmic increase in the cure rate compared with contemporaneous dactinomycin chemotherapy.

We next designed a series of phase III trials to answer clinically relevant questions. The first study addressed whether we could reduce the significant neuromuscular and myelosuppressive toxicity of vinblastine by reducing the dosage from 0.4 to 0.3 $\mathrm{mg} / \mathrm{kg}$ and still maintain therapeutic efficacy. As expected, the lower dose of vinblastine was associated with a reduction in toxicity, but the complete remission and cure rates were similar with the two arms.

Our subsequent phase III PVB study challenged one of the basic tenets of oncology, the utilization of maintenance therapy to prevent relapse. Patients achieving a disease-free status with PVB in this trial were randomized to a standard arm of 21 months of maintenance vinblastine (total of 2 years of chemotherapy) versus an experimental arm of just 12 weeks of PVB with no further therapy. One hundred thirteen patients entered this study at Indiana University or participating institutions in the Southeastern Cancer Study Group. The relapse rate was only $5 \%$, with or without maintenance vinblastine (6). This randomized clinical trial benefited patients by reducing duration of therapy and toxicity as well as lessening the financial expenditure of treatment.

†PVB: cisplatin, $20 \mathrm{mg} / \mathrm{m}^{2}$ days 1 to 5 ; vinblastine, $0.2 \mathrm{mg} / \mathrm{kg}$, days $1-2$; bleomycin, 30 units, days 2,9 , and 16. (i) Courses were repeated every 3 weeks for four courses; (ii) after PVB induction, single-agent maintenance vinblastine $(0.3 \mathrm{mg} / \mathrm{kg})$ every 4 weeks was utilized for a total of 24 mo of chemotherapy.

\section{PVB Versus Cisplatin + Etoposide + Bleomycin (BEP)}

Etoposide (VP-16) is an epipodophyllotoxin derivative with demonstrated single-agent activity in refractory testicular cancer (7). In 1978, we began our initial salvage chemotherapy studies with cisplatin + etoposide in patients who were not cured with PVB or similar induction therapy. Schabel et al. demonstrated remarkable synergism with cisplatin + etoposide in numerous preclinical models (8). We confirmed the remarkable synergism of these two active agents, as we achieved a $25 \%$ cure rate in patients who were not cured with their initial cisplatin combination chemotherapy (9). This represented the first time an adult solid tumor was cured with second-line chemotherapy.

From 1981 through 1984, the Southeastern Cancer Study Group conducted a randomized prospective study comparing PVB and BEP as initial induction chemotherapy (10), based on the promising results of cisplatin + etoposide as second-line therapy and the expectation for reduced neuromuscular toxicity with etoposide compared with vinblastine. No maintenance therapy was given in either arm. Postchemotherapy residual disease was resected if anatomically feasible. Two additional courses of the original induction regimen were given, deleting bleomycin, if carcinoma was found in the resected specimen.

A total of 244 patients from 24 institutions entered this trial. Of 121 patients treated with PVB, $74(61 \%)$ had a complete remission (CR), and another $15(13 \%)$ became disease-free after resection of teratoma (10 patients) or carcinoma (five patients). Among the 123 patients given BEP, 74 (60\%) had a CR, and 28 $(23 \%)$ became free of disease after resection of teratoma $(22$ patients) or carcinoma ( 6 patients). Thus, $74 \%$ became diseasefree after treatment with PVB and $83 \%$ after BEP. In the subgroup of advanced disseminated disease, there was a survival advantage for $\operatorname{BEP}(P=0.02)$.

Granulocytopenic toxicity, including granulocytopenic fever, was similar in the two arms. There was a major reduction in neuromuscular toxicity, as manifested by paresthesia, abdominal cramps, ileus, and myalgias, favoring the BEP arm. This was significant not only statistically, but also clinically. On the basis of this study, which demonstrated a reduction in morbidity and superior survival, we have used BEP since 1984 as first-line therapy for disseminated testicular cancer and have abandoned PVB.

\section{Subsequent Studies with BEP}

Good Risk (Minimal-Moderate Disease). Several groups have designed staging systems that attempt to discriminate good-risk from poor-risk disease $(11,12)$. This would allow the study of less toxic regimens in good-risk disease and more aggressive therapy in those validated to have poor-risk testicular cancer. We began a phase III study in 1984, completed in 1987, evaluating the standard four courses of BEP versus three courses ( 9 weeks) of $\mathrm{BEP}$ in good-risk disease. One hundred eighty-four patients entered this study, and $97 \%$ achieved a NED status confirming the accuracy of this definition of "good risk." An identical $92 \%$ of patients on each arm are continuously NED (13). We have updated this study for the 118 patients entered at Indiana University (median followup 9 years). There remain no differences between the two arms, with only four deaths in each arm. Furthermore, for patients with serum human chorionic gonadotropin less than 1,000 milliunits/ml, there were only two deaths out of 104 patients (14). Patients with good-risk metastatic disease (e.g., small pulmonary metastases) have a $98 \%$ cure rate with 9 weeks of chemotherapy, superior to surgical cure rates for most clinically localized cancers. Therefore, we have greatly ameliorated the toxicity and reduced the duration of therapy from the original PVB study in 1974 to the completion of this clinical trial in 1987. We have demonstrated that we could reduce vinblastine dosage, eliminate 20 months of maintenance vinblas- 


\section{Table 1. Poor-risk disease (all nonseminomatous patients)}

Lactic dehydrogenase $>10$ times upper limit of normal; human chorionic gonadotropin $>50,000$ units $/ \mathrm{ml}$, or alphafetoprotein $>10,000 \mathrm{ng} / \mathrm{ml}$

Any primary mediastinal nonseminomatous germ cell tumor Nonpulmonary visceral metastases (bone, liver, brain, etc.)

tine, substitute etoposide for vinblastine with improved efficacy and decreased toxicity, and finally, delete the fourth and final course of BEP in good-risk patients. This resulted in the elimination of the most toxic of the four courses because of potential for cumulative toxicities (bleomycin pulmonary fibrosis, cisplatin neurotoxicity, and ototoxicity). Also, during the years of this phase III study (1984-1987), we did not have availability of effective antiemetics, and the cumulative toxicities of anorexia, nausea, and vomiting were also a formidable challenge for patients with their fourth courses of BEP.

A larger European Organization for Research and Treatment of Cancer phase III study randomized 812 good-risk patients to $\mathrm{BEP} \times 3$ versus $\mathrm{BEP} \times 3$ with a fourth course of $\mathrm{EP}$. This study was performed to attempt to detect a $5 \%$ difference favoring four courses. The results were again identical, with $90.4 \%$ (three courses) versus $89.4 \%$ progression-free survival (15).

Definition of Poor-Risk (Advanced) Disease. Poor-risk disease consists of a more heterogeneous patient population and has been defined based on tumor markers, volume of metastatic disease, and multiplicity of anatomic sites $(11,12)$. These patients will have a $40-60 \%$ cure rate with standard therapy. In this group, the impetus has been to evaluate more aggressive chemotherapy in phase II and III studies to try to improve the therapeutic outcome.

An international group was convened to develop a consensus classification for poor-risk (and good-risk) germ cell tumors. Data were available on 5,862 patients, with a median followup time of 5 years. Only $14 \%$ of these patients comprised the poor-risk category, with a $41 \%$ 5-year disease-free survival and overall $48 \%$ 5-year survival (16). The cure rate with standard BEP is probably $10 \%$ higher, because the intergroup consensus included older regimens that did not include etoposide. This new definition (Table 1) is now incorporated in the current American intergroup trial for poor-risk disease.

Advanced Disease. The National Cancer Institute in the United States randomized poor-risk patients to PVB versus an experimental regimen that used double-dose $\left(40 \mathrm{mg} / \mathrm{m}^{2} \times 5\right)$ cisplatin. This phase III study revealed a statistically significant diseasefree and overall survival favoring the double-dose cisplatin arm (17). This study seemed to validate the concept of dose intensity. However, there were several flaws in the study methodology. First, there was a very small sample size $(n=57$ with a $2: 1$ randomization). Second, and most important, the experimental arm also included a fourth drug, etoposide, with PVB. BEP had superior survival compared with PVB in advanced poor-risk disease, with standard-dose cisplatin on both arms (10). Therefore, we designed a study in advanced disease in which the only

1. Li, M. C., Whitmore, W. F., Golbey, R. \& Grabstald, H. (1960) J. Am. Med. Assoc. 174, 145-153.

2. Samuels, M. L., Lanzotti, V. J., Holoye, P. Y., Boyle, L. E., Smith, T. L. \& Johnson, D. E. (1976) Cancer Treat. Rev. 3, 185-204.

3. Rosenberg, B., VanCamp, L. \& Krigas, T. (1965) Nature (London) 205, 678-699.

4. Higby, D. J., Wallace, H. J., Albert, D. J. \& Holland, J. F. (1974) Cancer 33, 1219-1225.

5. Einhorn, L. H. \& Donohue, J. P. (1977) Ann. Int. Med. 87, 293-298.

6. Einhorn, L. H., Williams, S. D., Troner, M., Greco, F. A. \& Birch, R. (1981)

N. Engl. J. Med. 305, 717-731. variable was the cisplatin dosage $\left(20 \mathrm{mg} / \mathrm{m}^{2} \times 5\right.$ versus $40 \mathrm{mg} / \mathrm{m}^{2}$ $\times 5)$ with BEP in both arms. One hundred fifty-three patients were evaluable. As expected, patients receiving double-dose cisplatin experienced considerably more toxicity than standard BEP. Unfortunately, there was no evidence of therapeutic superiority for the high-dose cisplatin arm, with $62.2 \%$ continuously NED with high-dose and $63.6 \%$ with standard BEP (18).

Salvage Therapy. Our concept for salvage therapy has always been to use cisplatin plus other active agents not previously used, as long as there was no progression during cisplatin combination chemotherapy. Cisplatin + etoposide was initiated in 1978 as salvage therapy after PVB (9). Our initial salvage therapy after $\mathrm{BEP}$ had been vinblastine $0.11 \mathrm{mg} / \mathrm{kg}$ day 1 and $2+$ ifosfamide 1.2 grams $/ \mathrm{m}^{2} \times 5+$ cisplatin $20 \mathrm{mg} / \mathrm{m}^{2} \times 5$ every 3 weeks for four courses. Between 1984 and 1989, 135 patients received this regimen as second-line therapy. Sixty-seven patients $(49.6 \%)$ achieved NED status, including $15(11 \%)$ who were NED after postchemotherapy resection of teratoma and $10(7.4 \%)$ NED after postchemotherapy resection of carcinoma. Thirtytwo $(23.7 \%)$ are continuously NED with minimal followup of 5 years (19).

High-dose therapy, with carboplatin and etoposide and autologous bone marrow transplant, was first started at Indiana University in 1986. Initially, this was used as a last attempt at curative therapy (third-line or later or after progression during cisplatin therapy). Six of these first 40 patients are $5+$ years continuously NED (20). We now use peripheral stem cells and granulocyte colony-stimulating factor, and we are able to safely administer carboplatin $700 \mathrm{mg} / \mathrm{m}^{2} \times 3+$ etoposide $750 \mathrm{mg} / \mathrm{m}^{2}$ $\times 3$. We currently use this therapy as initial salvage chemotherapy. Thirty-five of $65(54 \%)$ are continuously disease-free for a minimum of 2 years with this approach (21), demonstrating the remarkable chemosensitivity and curability of testicular cancer. Over $70 \%$ of patients with metastatic testicular cancer will be cured with BEP. Approximately half of patients who are not cured with their initial chemotherapy will still be cured with high-dose chemotherapy.

Future Studies. Testicular cancer has been a model for a curable neoplasm. We are currently exploring whether there are molecular as well as clinical differences that separate curable from incurable disease. We will evaluate specific poor-risk subsets, including salvage chemotherapy patients, primary mediastinal nonesminomatous germ cell tumors (22), transformation of teratoma to primitive neuroectodermal tumors (23), and laterelapse patients (24). This latter group is particularly intriguing. Metastatic testicular cancer grows very rapidly and is uniquely curable with cisplatin combination chemotherapy. However, $2-3 \%$ of patients initially diagnosed with testicular cancer will experience a late relapse beyond 2 years of last therapy. Most late relapses occur beyond 5 years, with our latest relapse 32 years after chemotherapy. Patients with late relapse may respond to cisplatin combination chemotherapy but are rarely curable with chemotherapy alone. We hope to identify an important molecular mechanism and then be able to exploit it as a therapeutic target.

7. Fitzharris, B. M., Kaye, S. B., Saverymuttu, S., Newlands, E. S., Barrett, A., Peckham, M. J. \& McElwain, T. J. (1980) Eur. J. Cancer 16, 1193-1197.

8. Schabel, F. M., Jr., Trader, M. W., Laster, W. R., Jr., Corbett, T. H. \& Griswold, D. P., Jr. (1979) Cancer Treat. Rep. 63, 1459-1473.

9. Williams, S. D., Einhorn, L. H., Greco, F. A., Oldham, R. \& Fletcher, R. (1980) Cancer 46, 2154-2158.

10. Williams, S. D., Birch, R., Irwin, L., Greco, A., Loehrer, P. J. \& Einhorn, L. H. (1987) N. Engl. J. Med. 316, 1435-1440.

11. Birch, R., Williams, S. D., Cone, A., Einhorn, L. H., Roark, P., Turner, S. \& Greco, F. A. (1986) J. Clin. Oncol. 4, 400-407. 
12. Bosl, G. J., Geller, N. L., Cirrincione, C., Vogelzang, N. J., Kennedy, B. J., Whitmore, W. F., Jr., Vugrin, D., Scher, H., Nisselbaum, J. \& Golbey, R. B. (1983) Cancer Res. 43, 3403-3407.

13. Einhorn, L. H., Williams, S. D., Loehrer, P. J., Birch, R., Drasga, R., Omura, G. \& Greco, F. A. (1989) J. Clin. Oncol. 7, 387-391.

14. Saxman, S., Finch, D., Gonin, R. \& Einhorn, L. H. (1998) J. Clin. Oncol. 16, 702-706.

15. de Wit, R., Roberts, J. T., Wilkinson, P. M., de Mulder, P. H., Mead, G. M., Fossa, S. D., Cook, P., de Prijck, L., Stenning, S. \& Collette, L. (2001) J. Clin. Oncol. 19, 1629-1640.

16. International Germ Cell Cancer Collaborative Group (1997) J. Clin. Oncol. 15, 594-603.

17. Ozols, R. F., Ihde, D. C., Linehan, M., Jacob, J., Ostchega, Y. \& Young, R. C. (1988) J. Clin. Oncol. 6, 1031-1040.

18. Nichols, C. R., Williams, S. D., Loehrer, P. J., Greco, F. A., Crawford, E. D.,
Weetlaufer, J., Miller, M. E., Bartolucci, A., Schacter, L. \& Einhorn, L. H. (1991) J. Clin. Oncol. 9, 1163-1172.

19. Loehrer, P. J., Gonin, R., Nichols, C. R., Weathers, T. \& Einhorn, L. H. (1998) J. Clin. Oncol. 16, 2500-2504.

20. Broun, E. R., Nichols, C. R., Jacob, J., Ostchega, Y. \& Young, R. C. (1992)Ann. Intern. Med. 11, 7124-7128.

21. Bhatia, S., Cornetta, K., Broun, R., Nichols, C., Abnour, R. \& Einhorn, L. H. (2000) J. Clin. Oncol. 18, 3346-3351.

22. Ganjoo, K. N., Rieger, K. M., Kesler, K. A., Sharma, M., Heilman, D. K. \& Einhorn, L. H. (2000) Cancer 88, 1051-1056.

23. Ganjoo, K. N., Foster, R. S., Michael, H., Donohue, J. P. \& Einhorn, L. H. (2001) J. Urol. 165, 1514-1516.

24. Baniel, J., Foster, R. S., Gonin, R., Messemer, J. E., Donohue, J. P. \& Einhorn, L. H. (1995) J. Clin. Oncol. 13, 1170-1176. 